\title{
Uncertainty Quantification of a Flapping Airfoil with Stochastic Velocity Deviations Using the Response Surface Method
}

\author{
Liangyu Zhao, ${ }^{*}, 1,2$ and Xiaqing Zhang ${ }^{2}$
}

\author{
${ }^{I}$ Key Laboratory of Dynamics and Control of Flight Vehicle, Ministry of Education, Beijing Institute of Technology, \\ Beijing 100081, China \\ ${ }^{2}$ School of Aerospace Engineering, Beijing Institute of Technology, Beijing 100081, China
}

\begin{abstract}
A practical flapping wing micro air vehicle should have ability to withstand stochastic deviations of flight velocities. To design a flapping airfoil with this ability, it is necessary to evaluate the impacts of velocity deviations on the flapping performances numerically or analytically. In this paper, the responses of the time-averaged thrust coefficient and the propulsive efficiency with respect to a stochastic flight velocity deviation under Gauss distribution are numerically investigated using a classic Monte Carlo method. The response surface method is employed to surrogate the high fidelity CFD model to save computational cost. It is observed that both of the time-averaged thrust coefficient and the propulsive efficiency obey a Gauss-like but not the exact Gauss distribution. The effect caused by the velocity deviation on the timeaveraged thrust coefficient is larger than the one on the propulsive efficiency.
\end{abstract}

Keywords: Uncertainty quantification, flapping airfoil, velocity deviation, response surface method.

\section{INTRODUCTION}

The Flapping Wing Micro Air Vehicle (FWMAV), inspired by birds, bats, insects, fishes and whales, has been receiving more and more attention from military and civilian application domains, since the MAV was generally defined by the Defense Advanced Research Projects Agency (DARPA) in 1997 [1]. With the evolution of the research on academic [2-5], some practical FWMAVs were also fabricated in last decade. For example, an unconventional FWMAV shown as Fig. (1), was proposed by Jones and Platzer [6]. In this design, the thrust is generated by the backward flapping bi-wing with a $25 \mathrm{~cm}$ span and the lift is mainly provided by the forward stationary wing. The present work is closely related to this model.

From the perspective of practical engineering, a real outdoor FWMAV should be capable to resist the unpredictable perturbations of the flight condition, for example, the inherent gust in the urban environment. Lian and Shyy discovered that a flapping airfoil is gust resistant by smoothing out large velocity variations of the freestream in 2007 [7]. Gopalan found that the airfoil with pure pitching motion can resist the vertical gust better than the airfoil with pure plunging motion in 2008 [8]. In 2009, Lian investigated the effect of a periodic head-on gust on the flight performance of a 2D flapping airfoil by numerical method [9]. It is concluded that the flapping airfoil can effectively alleviate the gust fluctuation under certain kinematics. Very recently, Viswanath and Tafti investigated the impact of frontal gusts on a rigid wing and discovered that the instantaneous lift and thrust profiles are influenced by the effective angle of attack,

*Address correspondence to this author at the Office 14, School of Aerospace Engineering, Beijing Institute of Technology, Beijing 100081, China; Tel: +86-13810122023 (Mobile), +86-10-68912419(Office); Fax: +86-10-68911040; E-mail: zhaoly@bit.edu.cn which is a function of the local flapping velocity, the freestream velocity, and the geometric angle of attack [10]. They also observed that the lift and the thrust appear in a similar fashion for gusts applied during the downstroke, whereas they experience opposite behaviors during the upstroke [10]. Following their previous work, Viswanath and Tafti investigated the impact of frontal gusts on a rigid wing and a flexible wing and discovered reverse lift response between the rigid wing and the flexible wing [11]. Zhao and Yang studied the impacts of a stochastic velocity deviation on the time-averaged thrust coefficient and the propulsive efficiency using the non-intrusive polynomial chaos method, and they found that the velocity deviation under a normal distribution has larger influence on the time-averaged thrust coefficient [12].

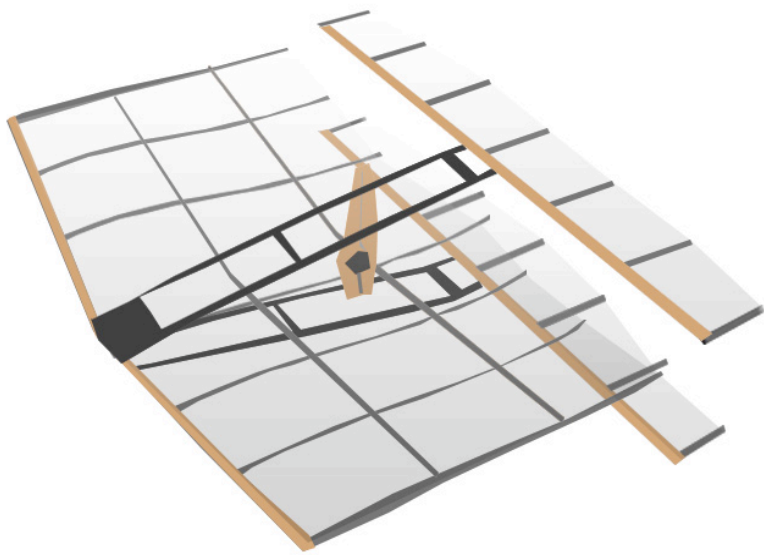

Fig. (1). Flapping wing MAV proposed by Jones and Platzer.

As an extension of our previous research [12], this paper focuses on investigating the impact of a velocity deviation on the aerodynamic performance perturbations of a flapping airfoil using a classic Monte Carlo method, while the 


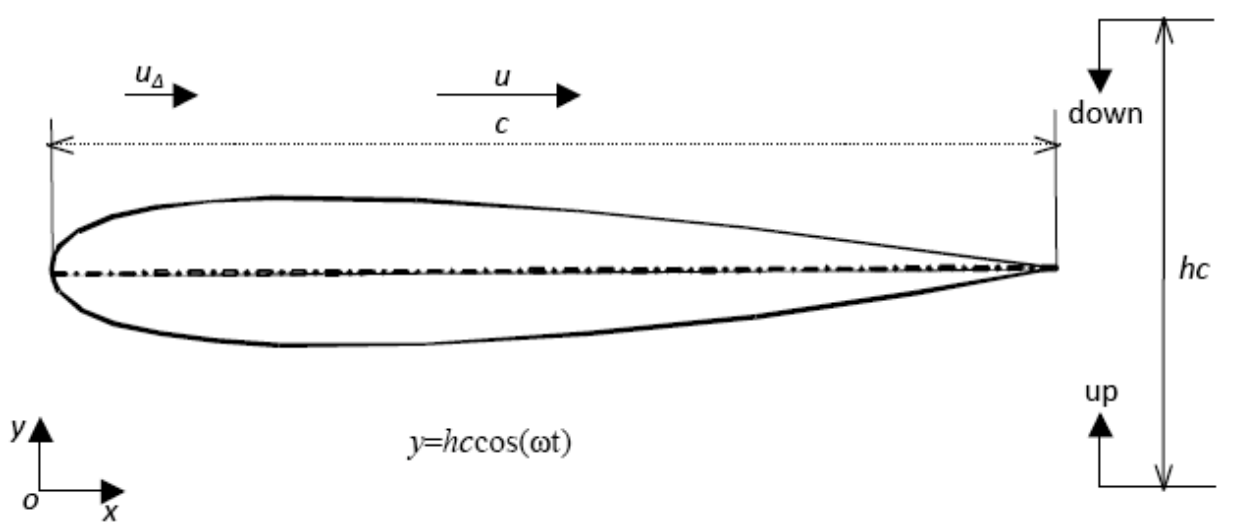

Fig. (2). Illustration of the flapping model in pure plunging motion.

previous research employed the non-intrusive polynomial chaos method as the uncertainty quantification technique. Furthermore, results obtained by these two distinct methods can be validated by each other. The velocity deviation is also assumed to obey a Gauss distribution, and the response surface method is employed to save computational cost.

\section{NUMERICAL METHOD TO EVALUATE FLAPP- ING PERFORMANCE}

To investigate the aerodynamic performance of a flapping airfoil numerically, an unsteady panel method [13], and Navier-Stokes equations computations [14] have been employed during past decade, especially the latter are used more and more widely with benefits of continuous improvements of computer sciences and Computational Fluid Dynamics (CFD) techniques $[15,16]$. In this study, a numerical method is employed to evaluate the flapping performance by solving unsteady Navier-Stokes equations with a dynamic mesh.

\section{Flapping Airfoil Model}

The real flapping model shown in Fig. (1) is a 3dimensional problem. However, it can be simplified to be a 2-dimensional flapping airfoil model with pure plunging motion. Under this simplification, the small-scale translation in flight direction and the trivial pitching motion along the leading edge are both ignored. The flapping motion, i.e., the pure plunging motion, is described using a harmonic function as equation (1),

$$
y(t)=h c \cos (\omega t)
$$

where $y(t)$ stands for the plunging motion, $c$ is the chord length, $h$ is the dimensionless plunging amplitude, $\omega$ is the angular frequency in $\mathrm{rad} / \mathrm{s}$. The reduced frequency, $k$, the Reynolds number, $R e$, and the Strouhla number, $S t$, are defined in equations (2) (4),

$$
\begin{aligned}
& k=2 \pi f c / u=\omega c / u \\
& R e=\rho u c / \mu \\
& S t=f A / u
\end{aligned}
$$

where $f$ is the flapping frequency in Hertz, $u$ is flow velocity of the far field, $\rho$ is the fluid density, $\mu$ is the fluid dynamic viscosity, $A$ is the wake width and can be estimated using the peak-to-peak excursion of the trailing edge, or more simply by twice the plunging amplitude. The simplified flapping model is illustrated in Fig. (2), where $u_{\Delta}$ is the velocity deviation probably induced by a constant wind in the flight direction.

The time-averaged thrust coefficient $\bar{C}_{T}$ and the power input coefficient $\vec{C}_{P}$ in one flapping cycle can be calculated by equation (5) and equation (6). Correspondingly, the propulsive efficiency is defined using equation (7),

$$
\begin{aligned}
& \bar{C}_{T}=-\frac{1}{T} \int_{t}^{t+T} C_{D}(t) d t \\
& \bar{C}_{P}=\frac{1}{T} \int_{t}^{t+T} C_{L}(t) \dot{y}(t) d t \\
& \eta=\bar{C}_{T} u / \bar{C}_{P}
\end{aligned}
$$

where $T$ is the period in seconds and $T=1 / f, \dot{y}(t)$ is the first order time derivation of $y(t) . C_{\mathrm{D}}$ and $C_{\mathrm{L}}$ are the timedependent drag coefficient and lift coefficient respectively. They are calculated by equations (8) (9),

$$
\begin{aligned}
& C_{D}=\frac{F_{x}}{0.5 \rho u^{2} S} \\
& C_{L}=\frac{F_{y}}{0.5 \rho u^{2} S}
\end{aligned}
$$

where $F_{x}$ and $F_{y}$ are the components of resulting aerodynamics force along horizontal (parallel with $u$ direction) and vertical (normal to $u$ direction) directions, $S$ is the reference area and equals to $c$ in value. For simplicity, we also use $C_{T}$ to stand for $\bar{C}_{T}$ in the next sections.

\section{Numerical Method}

The time-dependent drag coefficient and lift coefficient are evaluated by simulating the unsteady flow field around the flapping airfoil using CFD techniques. The commercial CFD package, FLUENT 6.3.26, is employed with assuming incompressible laminar flow. The mass and momentum equations were solved in a fixed inertial reference frame incorporating a dynamic mesh. These two governing equations are given by equations (10) and (11). 


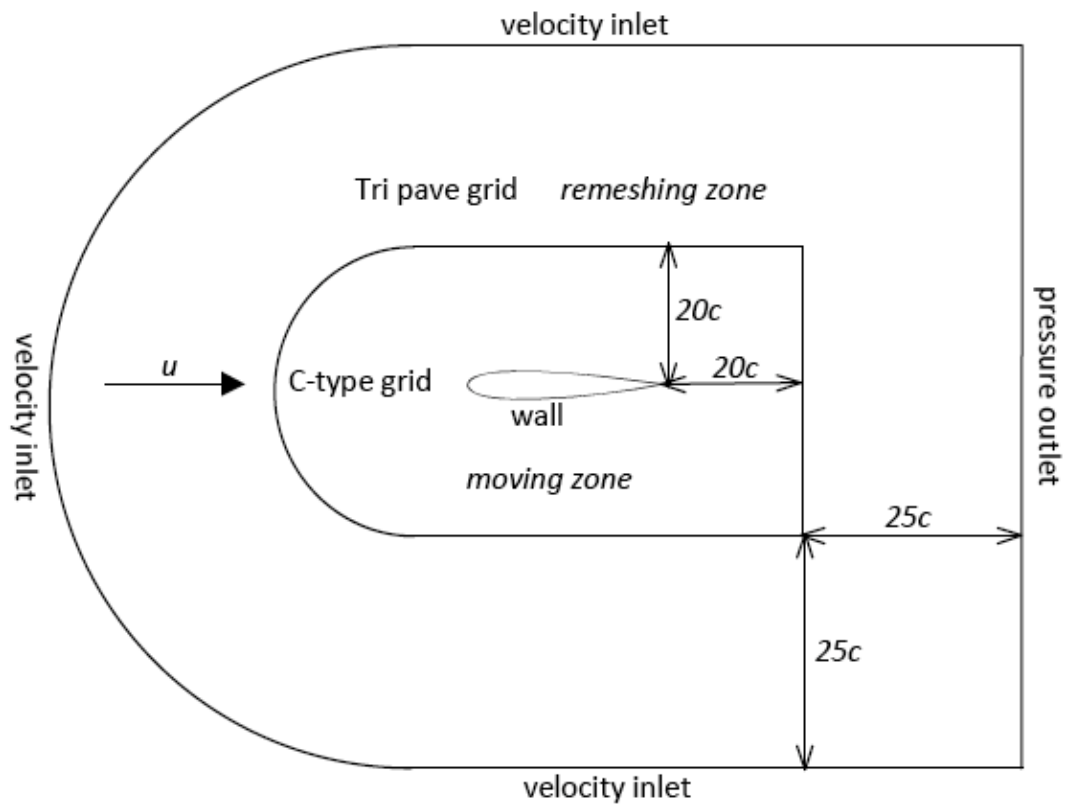

Fig. (3). Hybrid mesh topology with boundary conditions.

$\nabla \cdot \mathbf{u}=0$

$\rho\left(\frac{\partial \mathbf{u}}{\partial t}+\mathbf{u} \cdot \nabla \mathbf{u}\right)=-\nabla \mathbf{p}+\mu \nabla^{2} \mathbf{u}$,

where $\mathbf{u}$ and $\mathbf{p}$ are velocity and pressure, respectively.

The hybrid mesh which is shown schematically in Fig. (3) was employed to simulate the unsteady flow field. The computational domain is divided into two distinct zones: moving zone and re-meshing zone. The moving zone consists of C-type structured quadrilateral mesh, and the remeshing zone unstructured triangular mesh. The airfoil is located in the center of the computational domain, and the no-slip wall boundary condition is applied. The spacial scale of each zone and corresponding boundary condition are also shown in Fig. (3). The whole moving zone mesh, including the interfaces between these two zones, moves with the airfoil together according to the predefined motion. This means re-meshing only occurs at a distance of 20 to 45 reference lengths away from the airfoil body, which insures that the flow simulation around the airfoil is somewhat affected by the moving mesh. A representative C-type mesh in the very close neighborhood of the airfoil is shown in Fig. (4), and the grid size is $201 \times 101$ nodes (201 along every single airfoil surface, 101 in the vertical direction) with the thickness of the first layer grid around the airfoil equal to $0.0002 c$. The hybrid mesh was generated in GAMBIT v2.3.16.

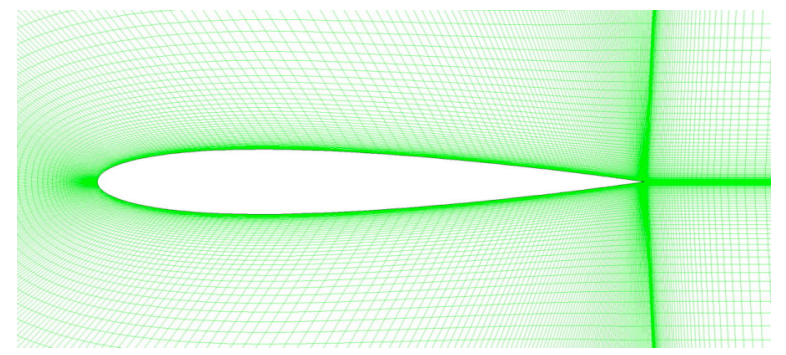

Fig. (4). C-type grid very close to the airfoil.
To validate the accuracy of the present approach, simulations were performed in 7 periods with 1000 and 500 time steps in one plunging period under the conditions of $k=2.0, h=0.4, u=34.7 \mathrm{~m} / \mathrm{s} \quad(M a=0.1), c=0.064 \mathrm{~m}$, and $R e=1.0 \times 10^{4}$ respectively. The coupling between the pressure and the velocity was achieved by means of the SIMPLE algorithm. Meanwhile, the discretizations of pressure and momentum terms were the Second Order scheme and the Second Order Upwind scheme. The time discretization was the First Order Implicit scheme, which is a more straightforward method in FLUENT for the dynamic mesh module [17]. The time variation of the plunging position and the time histories of $C_{D}$ in one period are shown in Fig. (5), with the comparisons with results obtained by Tuncer [18] and Miao [19]. These four results have a good agreement, though different mesh schemes were employed in these studies. Fig. (5) also shows that 500 time steps in one cycle are good enough to get concerned details. Based on these validations, $201 \times 101$ size grid with the first layer thickness of $0.0002 c$, and 500 time steps were employed for all of the next simulations. This numerical method was also validated and employed by our previous research $[12,20]$.

\section{Surrogate Model}

It takes about 24 hours to finish one evaluation by the above-mentioned numerical method on a personal computer. However, more than 100000 evaluations are needed to evaluate the probability distribution by the classic Monte Carlo method. The prohibited high computational cost makes us find a substitute to surrogate the high fidelity model. Surrogate models are a valuable and versatile tool for complex realistic engineering design [21]. The greatest benefit of a surrogate model is to save computational efforts with accepted approximation accuracy. Besides, a surrogate model is able to smooth the numerical noise of the high fidelity model effectively. In this research, the response surface method was employed as the approximation method, 


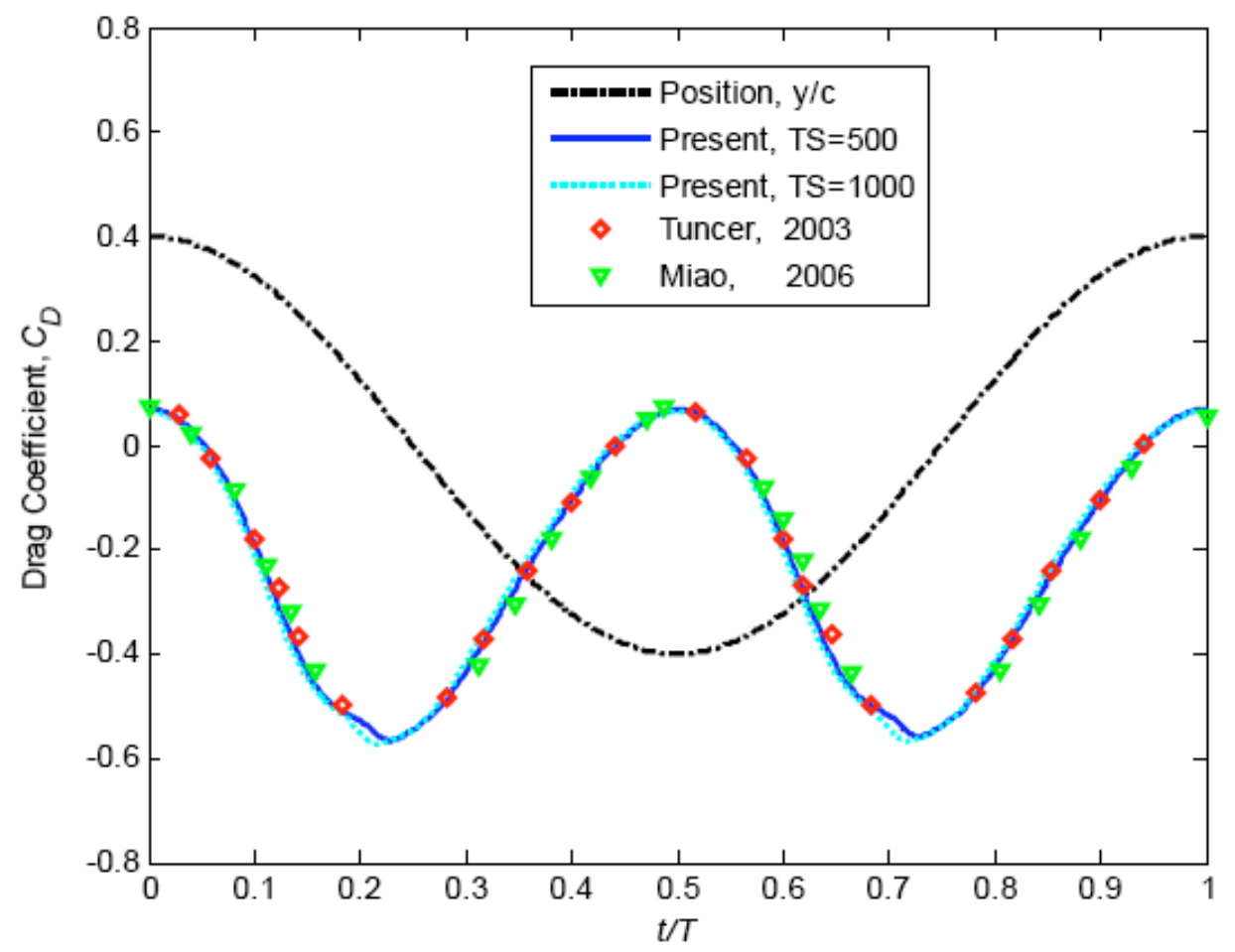

Fig. (5). Validation for the present numerical method.

and a nested and bounded sampling method as the design of experiments technique.

\section{CASE STUDY}

\section{Problem Definition}

The design condition is specified as $c=0.04 \mathrm{~m}, u=3.5 \mathrm{~m} / \mathrm{s}$, $f=30 \mathrm{~Hz}, h=0.375$, and the $R e=O(9000)$. It should be mentioned that these conditions are modified from the real flapping wing MAV shown in Fig. (1). The benchmark airfoil is NACA0012 airfoil. As a special case, the velocity derivation is assumed to obey a normal distribution in the range $[-1.01 .0] \mathrm{m} / \mathrm{s}$ with a mean value $0.0 \mathrm{~m} / \mathrm{s}$ and the standard derivation $0.20 \mathrm{~m} / \mathrm{s}$.

\section{Sampling Method}

Some evaluations based on high fidelity model should be done to construct the surrogate model. A sampling method is the way to decide the samples on which the time-averaged thrust coefficient and the propulsive efficiency are calculated by CFD techniques. The sampling method employed here is a nested and bounded sampling method which was proposed by Zhao and Yang [12]. The key feature of the sampling method is described as follows. The sampling method starts from 5 specified initial design points, which are the lower bound, upper bound, the point of mean value, the middle point of the lower bound and the mean value point, and the middle point of the upper bound and the mean value point. As the sampling process evolving, i.e., as the sampling index increasing, more and more samples are recruited in the specified sampling range. More details about this method how to evolve can be found in Zhao's paper [12]. When the index of the sampling is 10th, there are 35 samples, including 25 deterministic samples and 10 random samples.
These 35 samples and the time-averaged thrust coefficient and the propulsive efficiency related to these samples are shown in Table 1.

\section{Response Surface Method}

The Response Surface Method (RSM), also referred as Polynomial Regression Model, is a collection of mathematical and statistical techniques useful for the modeling and analysis of problems in which a response of interest is influenced by several variables [22]. However, the timeaveraged thrust coefficient and the propulsive efficiency are only affected by the velocity deviation in present problem. It is more exactly to call it polynomial regression model. The mathematical model of the response surface method with only one independent design variable is described as equation (12).

$y=\sum_{i=0}^{m} a_{i} x^{i}$,

where $y$ is the response, $x$ is the independent variable, $m$ is the polynomial order, $a_{i}$ is unknown coefficients and can be decided using the least square theory.

The changes of the Root Mean Square Errors (RMSEs) of the time-averaged thrust coefficient and the propulsive efficiency with increase of the orders of the involved polynomials are shown in Fig. (6). Further, the changes of the $\mathrm{R}$ square $\left(R^{2}\right)$ analysis with increase of the orders of the involved polynomials are shown in Fig. (7). Each of the regression models for the time-averaged thrust coefficient and the propulsive efficiency is good enough when the order of regression model is larger than 3rd. However, both of the polynomials orders for the time-averaged thrust coefficient and the propulsive efficiency are 9th in present study. These 
Table 1. Samples and Related Time-Averaged Thrust Coefficient and Propulsive Efficiency

\begin{tabular}{|c|c|c|c|}
\hline No. & Velocity Deviation & Time-averaged Thrust Coefficient & Propulsive Efficiency \\
\hline 1 & 1.000000 & 0.13517895 & 0.18608888 \\
\hline 2 & 0.000000 & 0.22150245 & 0.16762355 \\
\hline 3 & -1.000000 & 0.46104634 & 0.13166165 \\
\hline 4 & -0.500000 & 0.30739966 & 0.15335772 \\
\hline 5 & 0.500000 & 0.16974853 & 0.17754400 \\
\hline 6 & -0.250000 & 0.25886933 & 0.16189726 \\
\hline 7 & 0.250000 & 0.19296059 & 0.17275213 \\
\hline 8 & 0.100561 & 0.20885441 & 0.16969742 \\
\hline 9 & -0.750000 & 0.37637532 & 0.14662544 \\
\hline 10 & 0.750000 & 0.15123546 & 0.18135316 \\
\hline 11 & -0.569623 & 0.32285822 & 0.15150120 \\
\hline 12 & -0.125000 & 0.23897695 & 0.16482908 \\
\hline 13 & 0.125000 & 0.20612784 & 0.17028936 \\
\hline 14 & 0.331471 & 0.18468204 & 0.17409091 \\
\hline 15 & -0.375000 & 0.27980139 & 0.15870333 \\
\hline 16 & 0.375000 & 0.18033017 & 0.17479894 \\
\hline 17 & 0.609741 & 0.16103205 & 0.17941029 \\
\hline 18 & -0.625000 & 0.33524026 & 0.15004057 \\
\hline 19 & 0.625000 & 0.15987901 & 0.17963253 \\
\hline 20 & -0.831128 & 0.39654937 & 0.14427963 \\
\hline 21 & -0.875000 & 0.41749184 & 0.14146430 \\
\hline 22 & 0.875000 & 0.14275161 & 0.18414833 \\
\hline 23 & -0.700435 & 0.35858434 & 0.14864471 \\
\hline 24 & -0.062500 & 0.22987953 & 0.16630128 \\
\hline 25 & 0.062500 & 0.21322662 & 0.16880346 \\
\hline 26 & 0.629400 & 0.15953689 & 0.17967636 \\
\hline 27 & -0.187500 & 0.24843344 & 0.16329389 \\
\hline 28 & 0.187500 & 0.19959315 & 0.17159513 \\
\hline 29 & 0.826800 & 0.14573842 & 0.18323252 \\
\hline 30 & -0.312500 & 0.26935701 & 0.16041232 \\
\hline 31 & 0.312500 & 0.18658729 & 0.17380388 \\
\hline 32 & -0.443000 & 0.29397546 & 0.15563429 \\
\hline 33 & -0.437500 & 0.29280951 & 0.15589131 \\
\hline 34 & 0.437500 & 0.17486418 & 0.17624524 \\
\hline 35 & 0.264700 & 0.19135100 & 0.17296268 \\
\hline
\end{tabular}

two surrogate models are described in mathematics as equations (13) (14).

$$
\begin{aligned}
\hat{C}_{T}= & -0.0151 u_{t}^{9}+0.4579 u_{t}^{8}-6.1105 u_{t}^{7} \\
& +47.0146 u_{t}^{6}-229.5026 u_{t}^{5}+ \\
& 735.8300 u_{t}^{4}-1546.1366 u_{t}^{3}+2047.4037 u_{t}^{2}, \\
& -1545.2976 u_{t}+505.1111
\end{aligned}
$$

$$
\begin{aligned}
\hat{\eta}= & -0.0509 u_{t}^{9}+1.5753 u_{t}^{8}-21.5350 u_{t}^{7} \\
& +170.5246 u_{t}^{6}-861.7205 u_{t}^{5}+ \\
& 2880.9443 u_{t}^{4}-6369.9877 u_{t}^{3}+8978.7238 u_{t}^{2} \\
& -7317.7048 u_{t}+2626.1549
\end{aligned}
$$

where $u_{t}$ is the combination of the design velocity and the velocity deviation. In this case, i.e., when $m=9$, the RMSEs 


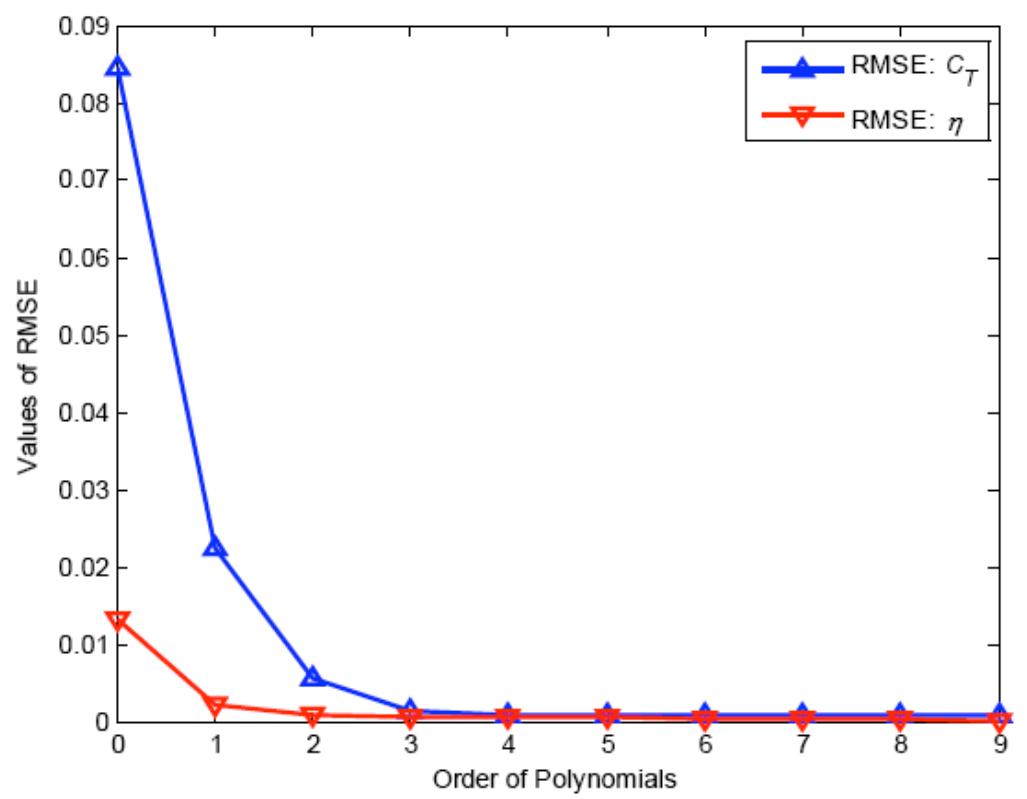

Fig. (6). The convergences of the RMSEs.

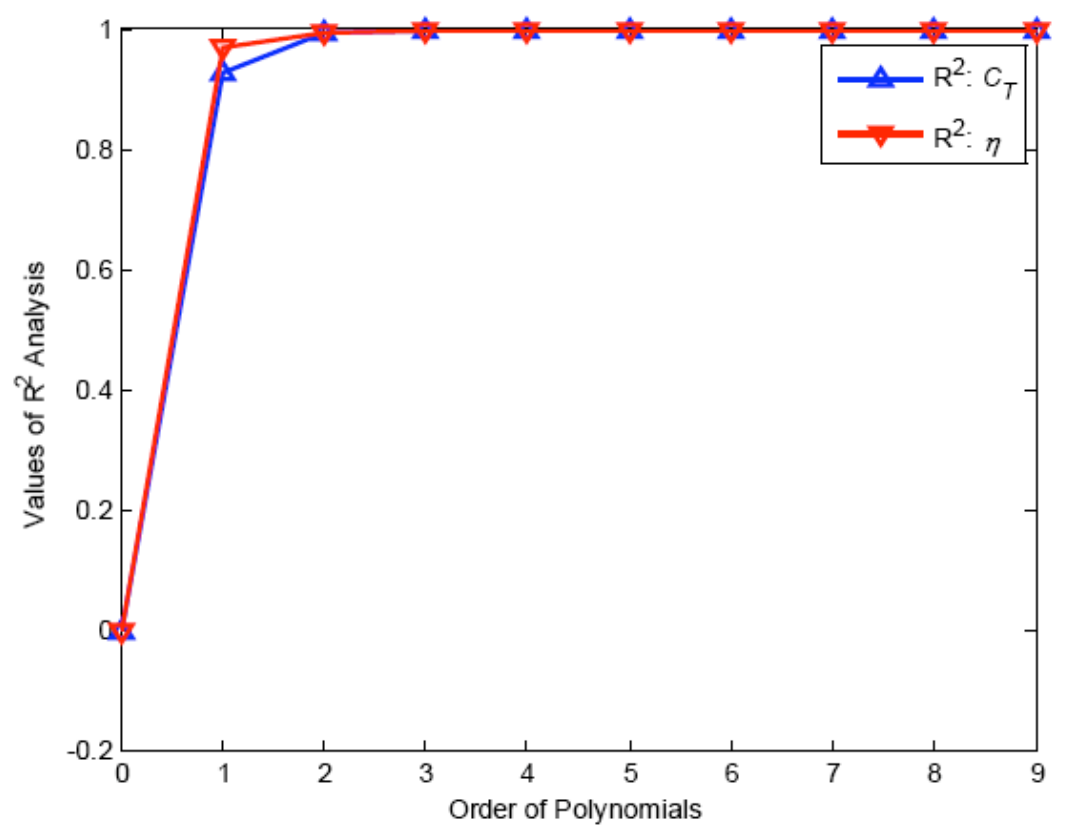

Fig. (7). The convergences of the R Square analysis.

of $C_{T}$ and $\eta$ are $9.5344 \mathrm{e}-4$ and $2.5175 \mathrm{e}-4$, and the coefficients of determination $\left(R^{2}\right)$ of $C_{T}$ and $\eta$ are 0.9999 and 0.9996 , respectively.

\section{Results and Analysis}

One million random points were generated in the range of $\left[\begin{array}{ll}-1.0 & 1.0\end{array}\right]$ under the normal distribution with the mean value $0.0 \mathrm{~m} / \mathrm{s}$ and the standard deviation $0.20 \mathrm{~m} / \mathrm{s}$. Here, it is assumed that one million samples for the classic Monte Carlo method are enough to obtain an accurate probability distribution. All of the responses were calculated by surrogate models, i.e., equation (13) and equation (14). The mean value and the standard deviation of the time-averaged thrust coefficient are 0.2242 and 0.0269 , while the mean value and the standard deviation of the propulsive efficiency are 0.1674 and 0.0044 . It is observed that the standard deviation of $C_{T}$ is larger than the standard deviation of $\eta$ by about 5 times, while the mean value of $C_{T}$ and $\eta$ are similar magnitude. This means that the time-averaged thrust coefficient is much more sensitive to the velocity deviation than the propulsive efficiency to the velocity deviation. Actually, this observation matches the conclusion of our previous study [12]. The histograms of the time-averaged thrust coefficient and an exact Gauss distribution with mean value 0.2242 and standard deviation 0.0269 are shown in Fig. (8). Furthermore, the histograms of the propulsive efficiency and an exact Gauss distribution with mean value 0.1674 and standard deviation 0.0044 are shown in Fig. (9). It is observed that both of probability distributions of the time-averaged thrust coefficient and the propulsive efficiency are Gauss-like but not the exact Gauss distribution. 


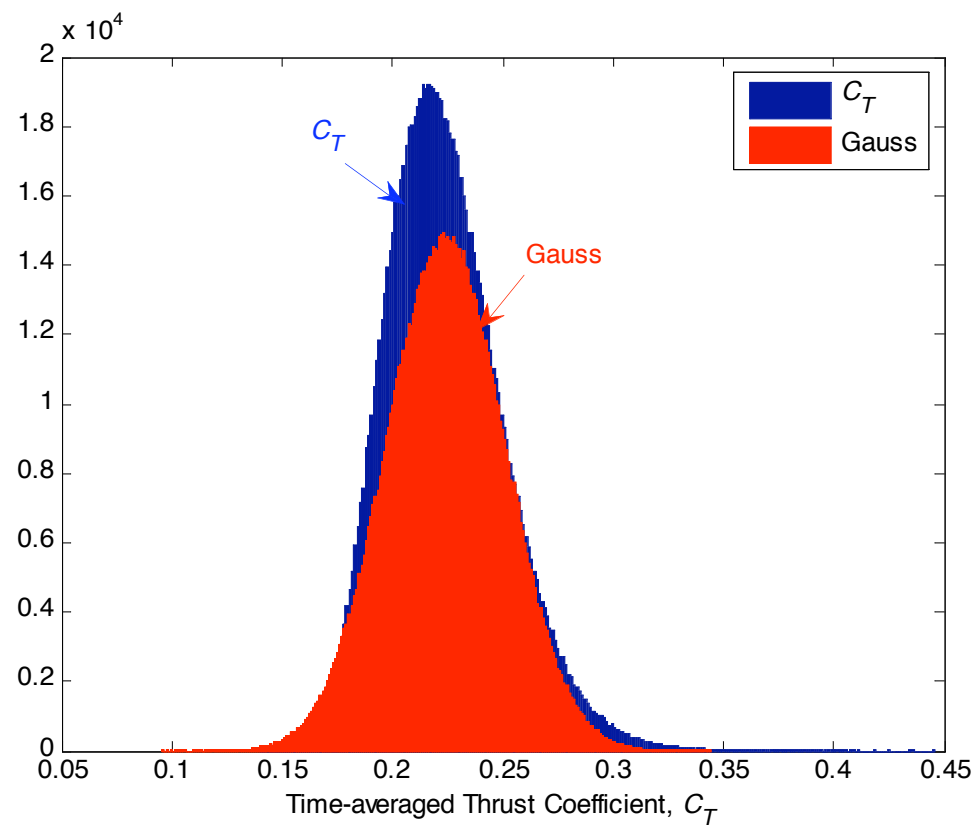

Fig. (8). Histogram of the time-averaged thrust coefficient.

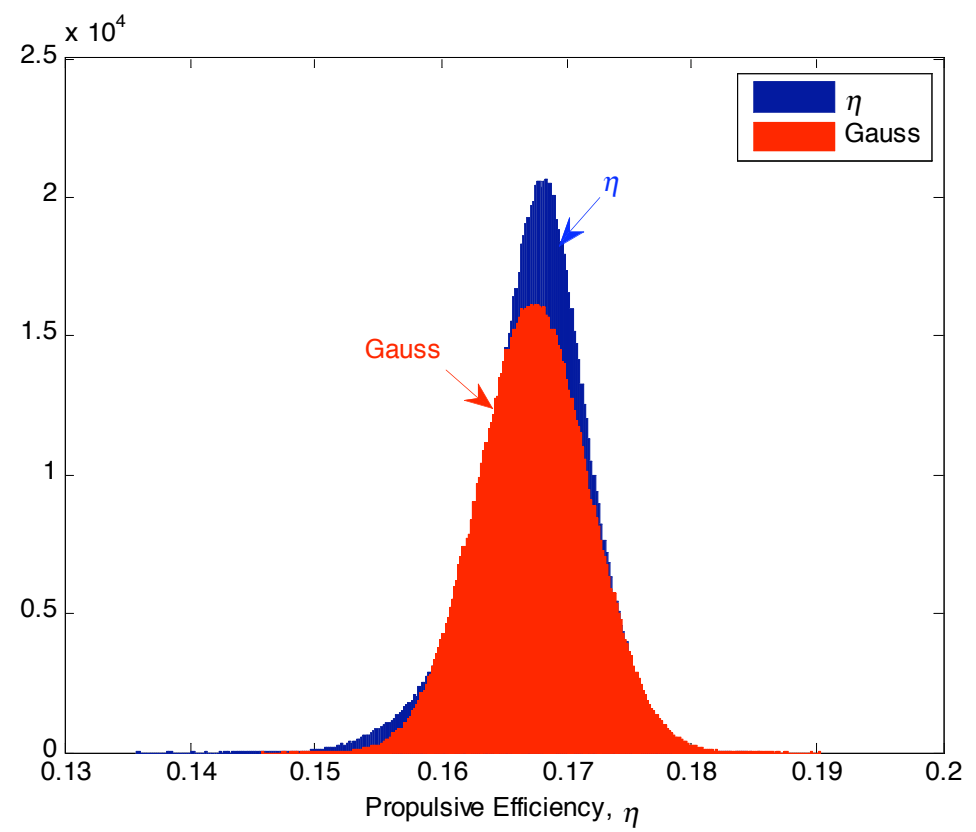

Fig. (9). Histogram of the propulsive efficiency.

\section{CONCLUSIONS}

The impacts of a velocity deviation on the time-averaged thrust coefficient and the propulsive efficiency of a flapping airfoil were numerically investigated using a classic Monte Carlo method. In the case of high fidelity model, the aerodynamic performance was analyzed by solving the unsteady Navier-Stokes equations with a dynamic mesh. A high order polynomial approximation model is constructed to surrogate the high fidelity model to save computational cost. In the case of the velocity deviation obeying normal distribution, it is observed that both of the time-averaged thrust coefficient and the propulsive efficiency obey Gauss-like but not the exact Gauss distribution. It is also observed that the time- averaged thrust coefficient is much more sensitive to the velocity deviation than the propulsive efficiency to the velocity deviation.

This work could be a preparation to the robust design of a flapping wing with respect to stochastic flight conditions.

\section{ACKNOWLEDGEMENTS}

This research was supported by the National Science Foundation for Post-doctoral Scientists of China (20090460216) and the Natural Science Foundation of China (10972034). 


\section{REFERENCES}

[1] J.M. McMichael and M.S. Francis, "Micro air vehicles - toward a new dimension in flight", DARPA Report, Arlington, USA, 1997.

[2] W. Shyy, M. Berg and D. Ljungqvist, "Flapping and flexible wings for biological and micro air vehicles", Prog. Aerosp. Sci., vol. 35, no. 5, pp. 455-505, 1999.

[3] S. P. Sane, "The aerodynamics of insect flight", J. Exp. Biol., vol. 206, no. 23, pp. 4191-4208, 2003.

[4] M. F. Platzer, K. D. Jones, J. Young,and J. C. S. Lai, "Flappingwing Aerodynamics: progress and challenges", AIAA J., vol. 46, no. 9, pp. 2136-2148, 2008.

[5] W. Shyy, H. Aono, S. K. Chimakurthi, P. Trizila, C.-K. Kang, C.E.S. Cesnik, and H. Liu, "Recent progress in flapping wing aerodynamics and aeroelasticity," Prog. Aerosp. Sci., vol. 46, no. 7, pp. 284-327, 2010.

[6] K.D. Jones and M.F. Platzer, "Bio-Inspired design of flapping wing micro air vehicles - An engineer's perspective", AIAA-2006-0037, in 44th AIAA Aerospace Sciences Meeting and Exhibit, Reno, Nevada, 9-12 January, 2006.

[7] Y. Lian and W. Shyy, "Aerodynamics of low Reynolds number plunging airfoil under gusty environment", AIAA 2007-0071, in 45th AIAA Aerospace Sciences Meeting and Exhibit, Reno, Nevada, 8-11 January, 2007.

[8] H. Gopalan and A. Povitsky, "Effect of frontal gusts on forward flapping flight", AIAA J., vol. 48, no. 9, pp. 2049-2062, 2010.

[9] Y. Lian, "Numerical study of a flapping airfoil in gusty environments", AIAA 2009-3952, in 27th AIAA Applied Aerodynamics Conference, San Antonio, Texas, 22-25 June, 2009.

[10] K. Viswanath and D. Tafti, "Effect of frontal gusts on forward flapping flight", AIAA J., vol. 48, no. 9, pp. 2049-2062, 2010.

[11] K. Viswanath and D. Tafti, "Effect of frontal gusts on flexible wings in forward flapping flight", AIAA-2010-4869, in 40th Fluid Dynamics Conference and Exhibit, Chicago, Illinois, 28 June-1 July, 2010.
[12] L. Zhao and S. Yang, "Uncertainty quantification for a flapping airfoil with a stochastic velocity deviation", in The 6th International Conference on Intelligent Unmanned Systems, Bali, Indonesia, 3-5 November, 2010.

[13] T. Cebeci, M. Platzer, H. Chen, K. Chang, and J. Shao, Analysis of Low-Speed Unsteady Airfoil Flows, Heidelberg: Springer, 2005.

[14] J. Young, S.M. Walker, R.J. Bomphrey, G.K. Taylor, and L.R. Thomas, "Details of insect wing design and deformation enhance aerodynamic function and flight efficiency", Science, vol. 325, no. 5947, pp. 1549-1552, 2009.

[15] N.S. Tachos, A.E. Filios, D.P. Margaris, and J.K. Kaldellis, "A computational Aerodynamics simulation of the NREL phase II rotor", Open Mech Eng J, vol. 3, pp. 9-16, 2009.

[16] A.M. Thomas and J.P. Abraham, "Numerical simulation of circular synthetic jets with asymmetric forcing profiles", Open Mech Eng $J$, vol. 4, pp. 1-7, 2010.

[17] T. Kinsey and G. Dumas, "Parametric study of an oscillating airfoil in a power-extraction regime", AIAA J., vol. 46, no. 6, pp. 13181330, 2008.

[18] I.H. Tuncer and M. Kaya, "Thrust generation caused by flapping airfoils in a biplane configuration”, J. Aircr., vol. 40, no. 3, pp. 509$515,2003$.

[19] J.M. Miao and M.H. Ho, "Effect of flexure on aerodynamic propulsive efficiency of flapping flexible airfoil”, J. Fluids Struct., vol. 22, pp. 401-41, 2006.

[20] L. Zhao and S. Yang, "Influence of thickness variation on the flapping performance of symmetric NACA airfoils in plunging motion", Math. Probl. Eng., vol. 2010, pp. 1-19, 2010.

[21] N.V. Queipo, R.T. Haftka, W. Shyy, T. Goel, R. Vaidyanathan, and P.K. Tucker, "Surrogate-based analysis and optimization", Prog. Aerosp. Sci., vol. 41, pp. 1-28, 2005.

[22] D. C. Montgomery, Design and Analysis of Experiments: Response Surface Method and Design, New Jersey: John Wiley and Sons, Inc., 2005.

(C) Zhao and Zhang; Licensee Bentham Open.

This is an open access article licensed under the terms of the Creative Commons Attribution Non-Commercial License (http: //creativecommons.org/licenses/by$\mathrm{nc} / 3.0 /$ ), which permits unrestricted, non-commercial use, distribution and reproduction in any medium, provided the work is properly cited. 